\title{
Does Discontinuing Teriparatide Treatment and Replacing It with Bisphosphonate Maintain the Volume of the Bone Fusion Mass after Lumbar Posterolateral Fusion in Women with Postmenopausal Osteoporosis?
}

\author{
Seiji Ohtori, Sumihisa Orita, Kazuyo Yamauchi, Yawara Eguchi, Yasuchika Aoki, Junichi Nakamura, \\ Miyako Suzuki, Gou Kubota, Kazuhide Inage, Yasuhiro Shiga, Koki Abe, Kazuki Fujimoto, \\ Hirohito Kanamoto, Masahiro Inoue, Hideyuki Kinoshita, Takeo Furuya, Masao Koda \\ Department of Orthopaedic Surgery, Graduate School of Medicine, Chiba University, Chiba, Japan
}

\section{Study Design: Retrospective case series.}

Purpose: The purpose of this study was to determine whether discontinuing teriparatide treatment and replacing it with bisphosphonate treatment maintains the volume of the fusion mass after posterolateral fusion (PLF) in women with postmenopausal osteoporosis. Overview of Literature: Clinical data support the efficacy of parathyroid hormone (PTH) for lumbar PLF. However, the use of PTH is limited to 2 years.

Methods: We treated 19 women diagnosed with osteoporosis and degenerative spondylolisthesis with teriparatide (20 $\mu \mathrm{g}$ daily subcutaneously). All patients underwent one-level instrumented PLF. Teriparatide was used during 2 months prior to surgery and more than 8 months after surgery. After discontinuing teriparatide treatment, all patients used bisphosphonate $17.5 \mathrm{mg}$ risedronate weekly, oral administration). Area of the fusion mass across the transverse processes at one segment was determined on an anteroposterior radiograph at 1,2, and 3 years after surgery.

Results: We followed 19 patients for 3 years. The average duration of teriparatide treatment was 11.5 months. The bone union rate was $95 \%$. The average area of the bone fusion mass was not significantly different between the right and left sides at 1, 2, or 3 years after surgery $(p>0.05)$.

Conclusions: This study showed that replacing teriparatide treatment with bisphosphonate maintained the bone fusion mass volume after PLF in women with postmenopausal osteoporosis.

Keywords: Lumbar; Spine; Fusion; Osteoporosis; Teriparatide; Surgery

\footnotetext{
Received Aug 26, 016; Revised Oct 1, 2016; Accepted Oct 5, 2016

Corresponding author: Seiji Ohtori

Department of Orthopaedic Surgery, Graduate School of Medicine, Chiba University,

1-8-1 Inohana, Chuo-ku, Chiba 260-8670, Japan

Tel: +81-43-226-2117, Fax: +81-43-226-2116, E-mail: sohtori@faculty.chiba-u.jp
} 


\section{Introduction}

Autologous iliac crest bone grafting, local bone, ceramics, demineralized bone matrix, and bone morphogenetic proteins (BMPs) are all methods used for lumbar fusion [1]. The use of BMPs increased significantly from 2004 to 2009 and $33 \%$ of the bone fusions performed in the United States in 2009 involved the use of BMPs [2]. Several authors have reported complications with autologous iliac crest bone grafting, such as donor site pain and fractures. BMPs have also been associated with disadvantages such as ectopic bone formation, vertebral osteolysis, and postoperative radiculitis in the lumbar spine [3].

Parathyroid hormone (PTH) has been recently used to increase vertebral bone mineral density (BMD) in osteoporotic patients $[4,5]$. Moreover, the efficacy of PTH for lumbar posterolateral fusion (PLF) has been reported using animal models [6-8]. Compared with an autograft only group, animal model experiments showed that the bone fusion rate after PTH treatment was significantly higher [6-8]. The efficacy of recombinant human PTH (134) (teriparatide) for fracture healing in the cervical spine was shown in a case report [9]. Further, delayed unions of type III odontoid fractures in three patients showed complete fusion after PTH treatment [9]. We previously reported that for instrumented PLF in the osteoporotic spine, daily subcutaneous injections of teriparatide with local bone grafting were more effective than local bone grafting alone [10]. Furthermore, the incidence of pedicle screw (PS) loosening in the teriparatide group was significantly lower than in the local bone grafting alone group in the osteoporotic spine [11]. In several Asian countries including Japan, BMPs are not approved for clinical use; thus, teriparatide is rapidly becoming the gold-standard treatment for bone fusion in osteoporotic spine surgery.

In a clinical setting, the use of PTH is limited to 2 years, and there is a possibility that the fusion mass will decrease or disappear once teriparatide is discontinued. However, no study has shown that cessation of teriparatide treatment will change the volume of the bone fusion mass after lumbar PLF in women with postmenopausal osteoporosis. Therefore, the purpose of the current study was to determine whether discontinuing teriparatide treatment, and replacing it with bisphosphonate treatment, maintains the bone volume of the fusion mass after PLF in women with postmenopausal osteoporosis.

\section{Materials and Methods}

\section{Patients}

Between January 2010 and January 2016, we retrospectively evaluated 19 patients treated at our university hospital. Patients were 65 to 79 years of age (mean \pm standard deviation [SD], 72.0 \pm 7.7 years). The main complaint was low back and leg pain, or leg pain only. All patients had lumbar degenerative spondylolisthesis (slipping of L3 or L4). Some patients showed intermittent claudication within 50 to $100 \mathrm{~m}$. Patients were diagnosed with one-level L3 or L4 degenerative spondylolisthesis, and spinal stenosis on radiographs, dynamic radiographs, and magnetic resonance imaging. Exclusion criteria were past lumbar surgery, spinal tumors, infections, or acute vertebral fractures of the lumbar spine. Diagnosis of spondylolisthesis and inclusion criteria for fusion surgery were (1) more than 5\% slip of the vertebra in a neutral position; or (2) translation greater than $3 \mathrm{~mm}$ between flexion and extension positions on radiographic evaluation. Informed consent was obtained from each of the participants. All of them were diagnosed as having osteoporosis based on the Japanese criteria [12]. BMD of the lumbar spine was measured before surgery. We evaluated pain according to the visual analog scale (VAS) score (0, no pain; 10 , worst pain) for low back pain and leg pain, and recorded Oswestry disability index for low back pain. Details of the patient background characteristics are shown in Table 1.

All patients underwent decompression and PLF at the

Table 1. Demographic characteristics

\begin{tabular}{lc} 
Characteristic & No. \\
No. of patients & 19 \\
\hline Sex (male:female) & $0: 19$ \\
\hline Age (yr) & $72.0 \pm 7.7(65-79)$ \\
\hline Symptom duration (mo) & $11.0(6-24)$ \\
\hline Follow-up after surgery (mo) & $36-60$ \\
\hline Bone mineral density (young adult mean) & $66.0 \pm 10.0(45-70)$ \\
\hline Pain score before surgery & \\
\hline Low back pain & $4.8 \pm 2.0$ \\
\hline$\quad$ Visual analogue scale & $40 \pm 16$ \\
\hline$\quad$ Oswestry disability index & \\
\hline & Leg pain \\
$\quad$ Visual analogue scale & $8.6 \pm 3.0$ \\
\hline
\end{tabular}


level of the spondylolisthesis. One-level PLF was performed using PSs and a local bone graft. Bilateral facet fusion was performed in all patients. The local bone consisted of the lamina from the decompression site and spinous process. We did not use other osteoconductive products for spinal fusion.

\section{Administration of teriparatide and risedronate}

Patients were administered teriparatide 2 months prior to surgery and more than 8 months after surgery, with average treatment duration of $11.5 \pm 2.5$ months (teriparatide $20 \mathrm{mg}$ by daily subcutaneous injection, Eli Lilly, Tokyo, Japan). We have previously reported that a longer period of teriparatide treatment ( $>6$ months before and after surgery) for bone union was more effective than a shorter period of treatment [13]. Thus, we used teriparatide 2 months before surgery and more than 8 months after surgery. Twelve patients used bisphosphonate (risedronate) (weekly oral administration of risedronate $17.5 \mathrm{mg}$, Eisai, Tokyo, Japan) for an average of $6.5 \pm 2.7$ months (range, 3-12 months) before using teriparatide. Seven patients did not use bisphosphonate before using teriparatide. After treatment with teriparatide, all patients used risedronate during the follow-up period (average, 30.6 \pm 15 months; range, 26-40 months).

\section{Radiographic evaluation}

Radiographs were used to evaluate bone union. Profile views of radiographic images in flexion and extension before and 1,2, and 3 years after surgery were evaluated. We classified bone union or failure to fuse at the surgical level. We defined bone union as less than $1.5^{\circ}$ of instability between the flexion and extension positions, or continuous fusion between transverse processes (Fig. 1). Evaluation of bone union was blinded and performed by three surgeons independently. Fusion was considered to have occurred if at least two of the observers agreed on this assessment. Sites that did not fused in 1 year were excluded from the follow-up examination.

Antero-posterior radiographic views were also evaluated at 1,2, and 3 years after surgery in all patients. The area of the bone fusion between transverse processes on each side was measured using a computer-linked digitizer. Three observers measured the bone volume, and the data were averaged.

\section{Statistical analyses}

A one-way analysis of variance with post hoc comparisons was used to evaluate follow-up data. A $p<0.05$ was considered statistically significant.

\section{Results}

Table 1 shows the demographic characteristics of the patients before surgery. Fusion rates were $84 \%, 95 \%$, and $95 \%$ at 1,2 , and 3 years after surgery (Table 2). One patient showed absence of fusion bilaterally 3 years after surgery. We evaluated the change of bone volume of the fusion mass in 32 segments of 16 patients who had evidence of fusion at 1 year after surgery.

Fig. 2 shows the average area of the bone fusion mass on the right and left sides. There was no significant difference in the average area of the bone fusion mass between sides. The average area of bone fusion was not significantly different after surgery for either side $(p>0.05)$ (right side at 1 year, $765.1 \pm 70.8 \mathrm{~mm}^{2} ; 2$ years, $865.1 \pm 80.4 \mathrm{~mm}^{2}$; and 3 years, $787.2 \pm 69.8 \mathrm{~mm}^{2}$; left side at 1 year, $933.2 \pm 78.2 \mathrm{~mm}^{2}$;
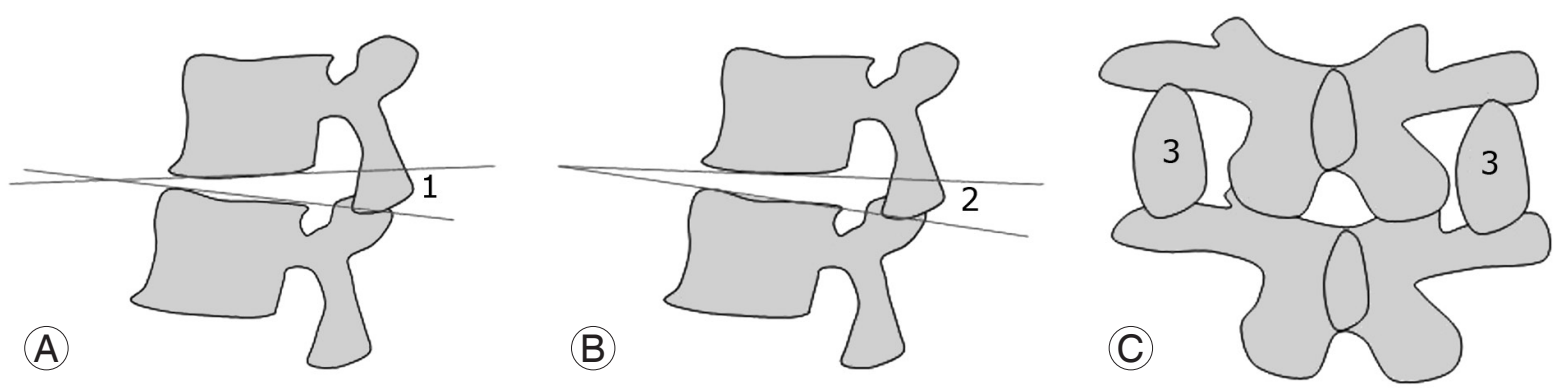

Fig. 1. Radiographic evaluation of bone fusion. Radiographs in flexion ( $\mathbf{A})$ and extension (B). We defined bone union as less than $1.5^{\circ}$ of instability between the flexion and extension positions (1-2), or continuous fusion between transverse processes (3 of C). 
Table 2. Number of fusion

\begin{tabular}{lcc} 
Status of fusion & 1 Year & 2 Year \\
\hline Total no. of patients & 19 & 19 \\
\hline No. of patients showing fusion & 16 & 18 \\
\hline $\begin{array}{l}\text { No. of segments (\%) (fusion across the transverse processes or } \\
\text { fusion by dynamic X-ray) }\end{array}$ & $32(84)$ & $36(95)$ \\
\hline Total no. of evaluated segments & 38 & $36(95)$ \\
\hline
\end{tabular}

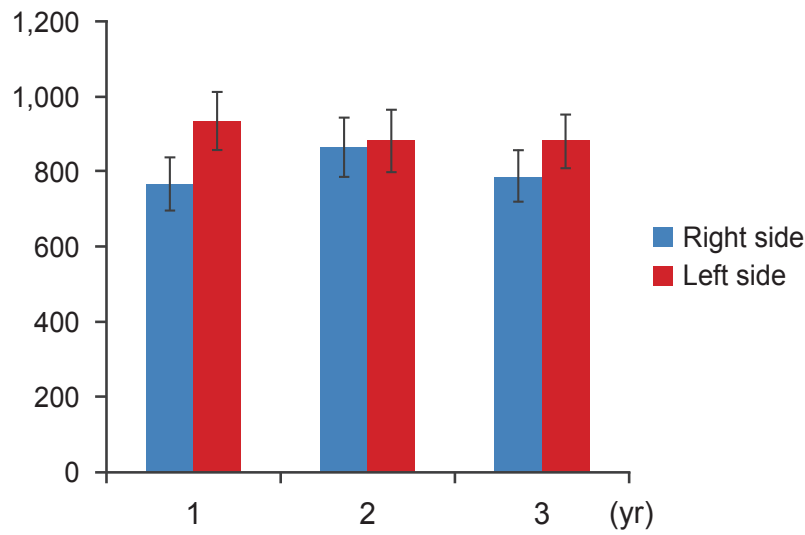

Fig. 2. Average area of bone fusion mass over time. There was no significant difference in average area of bone fusion mass between the right and left sides, nor was there a difference over time.

2 years, $880.6 \pm 84.3 \mathrm{~mm}^{2}$; and 3 years: $880.5 \pm 72.0 \mathrm{~mm}^{2}$ ).

\section{Discussion}

The current study showed that discontinuing teriparatide treatment did not reduce the volume of the bone fusion mass after PLF in women with postmenopausal osteoporosis within 3 years after surgery when a bisphosphonate was administered following teriparatide discontinuation.

In several Asian countries including Japan, BMPs are not available for spinal fusion. Thus, other agents are needed for spinal fusion in patients with osteoporosis. Bisphosphonates are a potential class of therapeutic agents for osteoporosis. Several animal studies have investigated the effects of bisphosphonates on spinal fusion. Fusion masses in alendronate-treated rats undergoing an intertransverse fusion using an autograft appeared radiographically larger and denser than those in control rats despite lower fusion rates [14]. Conversely, rabbits undergoing an inter-transverse process fusion with zoledronic acid treatment achieved increased fusion mass size and bone mineral content with increased fusion rate [15]. Only one prospective randomized trial has evaluated alendronate for lumbar interbody fusion [16]. Forty patients with osteoporosis, who were candidates for one-level posterior lumbar interbody fusion, were randomly assigned to an alendronate-treated group or a control group. A solid fusion was achieved in $95 \%$ of the patients in the alendronate-treated group compared with $65 \%$ of those in the control group [16].

Recently, subcutaneous injections of teriparatide $(20 \mu \mathrm{g} /$ day) have been used effectively for a maximum of 24 months to increase BMD in patients with osteoporosis. Compliance data from 1,671 patients showed that $71.9 \%$ of patients remained on teriparatide treatment after 12 months [17]. Bone formation biomarkers rapidly increased within 1 month of the initiation of teriparatide treatment, with smaller increases in resorption markers. After 12 months of teriparatide treatment, there was an increase in BMD at the lumbar spine, femoral neck, and total hip, and a decrease in the VAS score for back pain [17].

We previously reported on the efficacy of teriparatide for spinal fusion. Fifty-seven women with osteoporosis diagnosed with degenerative spondylolisthesis were divided into two treatment groups, a teriparatide group $(n=29$, daily subcutaneous injection of $20 \mu \mathrm{g}$ of teriparatide) and a bisphosphonate group $(n=28$, weekly oral administration of $17.5 \mathrm{mg}$ of risedronate) [10]. All patients underwent decompression and PLF with a local bone graft. Daily injection of teriparatide for bone union was more effective than oral administration of bisphosphonate [10]. Furthermore, a longer period of teriparatide treatment for bone union was more effective than a shorter period of treatment $[10,13]$.

Teriparatide effectively increases BMD, but its period of use is limited to 2 years. It is difficult to maintain the BMD gains achieved by teriparatide if the patients do not use bisphosphonates after discontinuing teriparatide [18,19]. Both hormone replacement therapy and bisphosphonates 
have been shown to maintain or increase BMD at the lumbar spine and hip after discontinuation of teriparatide treatment [18,19]. Muschitz et al. [20] demonstrated that the addition of alendronate 9 months after the initiation of teriparatide treatment resulted in an augmented increase in BMD both in the lumbar spine and hip region. In the alendronate combination group, with the exception of areal BMD and the trabecular component of femoral neck BMD, the increase in BMD was significantly greater compared with the teriparatide monotherapy group [20]. These results indicate that the use of bisphosphonate after discontinuation of teriparatide is necessary to maintain BMD. In the current study, after treatment with teriparatide, all patients used risedronate during the follow-up period, which prevented a reduction in the volume of the bone fusion mass 3 years after PLF in women with postmenopausal osteoporosis.

The current study has some limitations. First, it is a retrospective study based on a small sample. Second, we examined BMD in the lumbar spine before surgery, but we did not examine BMD after surgery. It would have been better to examine BMD after surgery, but were unable to so as we experienced instrumentation problems and could not get accurate BMD data. Third, we did not evaluate serum bone biomarkers. Fourth, we used risedronate after successful spinal fusion in all patents, but did not examine a control group without the use of risedronate. Fifth, we used radiography to evaluate bone union; however, we did not evaluate bone fusion using computed tomography.

\section{Conclusions}

In conclusion, the current study showed that after treatment with teriparatide, all women with postmenopausal osteoporosis who used risedronate during a 3-year followup period maintained bone fusion mass volume after PLF.

\section{Conflict of Interest}

No potential conflict of interest relevant to this article was reported.

\section{References}

1. Miyazaki M, Tsumura H, Wang JC, Alanay A. An update on bone substitutes for spinal fusion. Eur Spine J 2009;18:783-99.
2. Bae HW, Rajaee SS, Kanim LE. Nationwide trends in the surgical management of lumbar spinal stenosis. Spine (Phila Pa 1976) 2013;38:916-26.

3. McGovern SC, Fong W, Wang JC. Can bone morphogenetic protein binding peptide increase efficiency of bone formation? Spine (Phila Pa 1976) 2010;35:16559.

4. Dempster DW, Cosman F, Kurland ES, et al. Effects of daily treatment with parathyroid hormone on bone microarchitecture and turnover in patients with osteoporosis: a paired biopsy study. J Bone Miner Res 2001;16:1846-53.

5. Neer RM, Arnaud CD, Zanchetta JR, et al. Effect of parathyroid hormone (1-34) on fractures and bone mineral density in postmenopausal women with osteoporosis. N Engl J Med 2001;344:1434-41.

6. Lehman RA Jr, Dmitriev AE, Cardoso MJ, et al. Effect of teriparatide $[\operatorname{rhPTH}(1,34)]$ and calcitonin on intertransverse process fusion in a rabbit model. Spine (Phila Pa 1976) 2010;35:146-52.

7. O'Loughlin PF, Cunningham ME, Bukata SV, et al. Parathyroid hormone (1-34) augments spinal fusion, fusion mass volume, and fusion mass quality in a rabbit spinal fusion model. Spine (Phila Pa 1976) 2009;34:121-30.

8. Abe Y, Takahata M, Ito M, Irie K, Abumi K, Minami A. Enhancement of graft bone healing by intermittent administration of human parathyroid hormone (134 ) in a rat spinal arthrodesis model. Bone 2007;41: 775-85.

9. Rubery PT, Bukata SV. Teriparatide may accelerate healing in delayed unions of type III odontoid fractures: a report of 3 cases. J Spinal Disord Tech 2010; 23:151-5.

10. Ohtori S, Inoue G, Orita S, et al. Teriparatide accelerates lumbar posterolateral fusion in women with postmenopausal osteoporosis: prospective study. Spine (Phila Pa 1976) 2012;37:E1464-8.

11. Ohtori $S$, Inoue $G$, Orita $S$, et al. Comparison of teriparatide and bisphosphonate treatment to reduce pedicle screw loosening after lumbar spinal fusion surgery in postmenopausal women with osteoporosis from a bone quality perspective. Spine (Phila $\mathrm{Pa}$ 1976) 2013;38:E487-92.

12. Soen S, Fukunaga M, Sugimoto T, et al. Diagnostic criteria for primary osteoporosis: year 2012 revision. J Bone Miner Metab 2013;31:247-57. 
13. Ohtori S, Orita S, Yamauchi K, et al. More than 6 months of teriparatide treatment was more effective for bone union than shorter treatment following lumbar posterolateral fusion surgery. Asian Spine J 2015;9:573-80.

14. Huang RC, Khan SN, Sandhu HS, et al. Alendronate inhibits spine fusion in a rat model. Spine (Phila Pa 1976) 2005;30:2516-22.

15. Bransford R, Goergens E, Briody J, Amanat N, Cree A, Little D. Effect of zoledronic acid in an L6-L7 rabbit spine fusion model. Eur Spine J 2007;16:557-62.

16. Nagahama K, Kanayama M, Togawa D, Hashimoto T, Minami A. Does alendronate disturb the healing process of posterior lumbar interbody fusion? A prospective randomized trial. J Neurosurg Spine 2011;14:500-7.

17. Yamamoto T, Taketsuna M, Guo X, Sato M, Sowa H. The safety and effectiveness profile of daily teripara- tide in a prospective observational study in Japanese patients with osteoporosis at high risk for fracture: interim report. J Bone Miner Metab 2014;32:699-708.

18. Rittmaster RS, Bolognese M, Ettinger MP, et al. Enhancement of bone mass in osteoporotic women with parathyroid hormone followed by alendronate. J Clin Endocrinol Metab 2000;85:2129-34.

19. Kurland ES, Heller SL, Diamond B, McMahon DJ, Cosman F, Bilezikian JP. The importance of bisphosphonate therapy in maintaining bone mass in men after therapy with teriparatide [human parathyroid hormone(1-34)]. Osteoporos Int 2004;15:992-7.

20. Muschitz C, Kocijan R, Fahrleitner-Pammer A, Lung $\mathrm{S}$, Resch H. Antiresorptives overlapping ongoing teriparatide treatment result in additional increases in bone mineral density. J Bone Miner Res 2013;28: 196-205. 